\title{
DISTq: An Iterative Analysis of Glucose Data for Low-Cost, Real-Time and Accurate Estimation of Insulin Sensitivity
}

\author{
Paul D. Docherty ${ }^{1}$, J. Geoffrey Chase ${ }^{*}, 1$, Thomas Lotz ${ }^{1}$, Christopher E. Hann ${ }^{1}$, Geoffrey M. Shaw ${ }^{2}$, \\ Juliet E. Berkeley ${ }^{2}$, J.I. Mann ${ }^{3}$ and Kirsten McAuley ${ }^{3}$ \\ ${ }^{I}$ Department of Mechanical Engineering, University of Canterbury, New Zealand \\ ${ }^{2}$ Department of Medicine, Christchurch School of Medicine, University of Otago, Christchurch, New Zealand \\ ${ }^{3}$ Edgar National Centre for Diabetes Research, University of Otago, Dunedin, New Zealand
}

\begin{abstract}
Insulin sensitivity ( $S I)$ estimation has numerous uses in medical and clinical situations. However, highresolution tests that are useful for clinical diagnosis and monitoring are often too intensive, long and costly for regular use. Simpler tests that mitigate these issues are not accurate enough for many clinical diagnostic or monitoring scenarios. The gap between these tests presents an opportunity for new approaches.

The quick dynamic insulin sensitivity test (DISTq) utilises the model-based DIST test protocol and a series of population estimates to eliminate the need for insulin or C-peptide assays to enable a high resolution, low-intensity, real-time evaluation of SI. The method predicts patient specific insulin responses to the DIST test protocol with enough accuracy to yield a useful clinical insulin sensitivity metric for monitoring of diabetes therapy.

The DISTq method replicated the findings of the fully sampled DIST test without the use of insulin or C-peptide assays. Correlations of the resulting $S I$ values was $\mathrm{R}=0.91$. The method was also compared to the euglycaemic hyperinsulinaemic clamp (EIC) in an in-silico Monte-Carlo analysis and showed a good ability to re-evaluate $S I_{E I C}(\mathrm{R}=0.89)$, compared to the fully sampled DIST $(\mathrm{R}=0.98)$

Population-derived parameter estimates using a-posteriori population-based functions derived from DIST test data enables the simulation of insulin profiles that are sufficiently accurate to estimate $S I$ to a relatively high precision. Thus, costly insulin and C-peptide assays are not necessary to obtain an accurate, but inexpensive, real-time estimate of insulin sensitivity. This estimate has enough resolution for SI prediction and monitoring of response to therapy. In borderline cases, re-evaluation of stored (frozen) blood samples for insulin and C-peptide would enable greater accuracy where necessary, enabling a hierarchy of tests in an economical fashion.
\end{abstract}

\section{INTRODUCTION}

Insulin resistance $(I R)$ has been widely accepted as a risk factor for type 2 diabetes (T2DM) and cardiovascular disease. A long-term study has shown those who developed T2DM had an average initial $I R 60 \%$ higher than those who remained healthy [1]. An early diagnosis of $I R$ may prompt interventions with the potential to delay the onset of the disease and therefore reduce long-term complications and health costs. However, the high cost and/or low resolution of the available insulin sensitivity $(S I, S I=1 / I R)$ tests precludes widespread screening [2]. Thus many diagnoses are not made until later in the disease stage and limit the potential gains [3].

It is generally agreed that $S I$ is a measure of the efficiency of insulin for the reduction of glucose in the blood. However, the many available tests measure this effect in different ways $[4,5]$. It is therefore necessary for the researcher/clinician to decide which test is best for any given

*Address correspondence to this author at the Department of Mechanical Engineering, University of Canterbury, New Zealand;

E-mail: geoff.chase@canterbury.ac.nz situation in terms of intensity, cost, accuracy and physiological relevance. This decision thus implicitly states that there exists a hierarchy of such tests, each with different clinical use, and associated level of accuracy or resolution.

The hyper-insulinaemic euglycaemic clamp (EIC) [6] is widely accepted as the gold standard for SI testing. However, it is not used as a screening tool due to its high cost and clinical intensity. Tests such as the oral glucose tolerance test (OGTT), intravenous glucose tolerance test (IVGTT), and insulin-modified IVGTT (IM-IVGTT) are of lesser intensity, but equally lower accuracy and repeatability [7, 8]. The homeostatic model assessment (HOMA) or 2hr-OGTT are sufficiently inexpensive in cost, clinical time and intensity that they are regularly used for screening purposes. However, screening is not necessarily effective, because these tests have relatively poor resolution in the region of clinical significance $[7,9,10]$. They are thus even less effective in measuring the impact or change resulting from intervention. Table 1 summarises the accuracy and time demands of these $S I$ tests. 
Table 1. Overview of the Most Frequently Used Insulin Sensitivity Tests

\begin{tabular}{|c|c|c|c|c|c|c|}
\hline & \multicolumn{2}{|c|}{ Accuracy } & \multicolumn{2}{|c|}{ Time Demand (Hours) } & \multirow[b]{2}{*}{ Notes } & \multirow[b]{2}{*}{ References } \\
\hline & $\begin{array}{c}\text { Intra- } \\
\text { Subject CV }\end{array}$ & $\begin{array}{l}\text { Correlation to } \\
\text { Gold Standard }\end{array}$ & Clinician & Participant & & \\
\hline EIC & 4 to $10 \%$ & Gold standard & 10 & 4 & Results is not guaranteed with an inexperienced clinician & {$[5,6,11]$} \\
\hline IVGTT & $21 \%$ & 0.44 to 0.89 & $3-5$ & $3-5$ & Result generally requires a complex computerised solver & {$[8,12,13]$} \\
\hline OGTT & 7 to $15 \%$ & 0.6 to 0.8 & $3-4$ & $3-4$ & $\begin{array}{l}\text { Rate of appearance of glucose in plasma is relatively } \\
\text { unknown }\end{array}$ & {$[5,14,15]$} \\
\hline 2hr-OGTT & 15 to $40 \%$ & 0.45 to 0.74 & 0.5 & 2.5 & Poor repeatability in terms of re-classification rate & {$[7,14,16]$} \\
\hline HOMA & 10 to $40 \%$ & -0.6 to -0.9 & 0.25 & 0.25 & Low resolution in region of clinical significance & {$[5,17,18]$} \\
\hline
\end{tabular}

Shown are the assessed accuracies in coefficient of variation ( $\mathrm{CV}=\mathrm{SD} / \mathrm{mean})$ and correlation to the gold standard EIC; and the time demand in hours for the clinician and the participant.

A real-time and clinically relevant $S I$ metric could be of significant value in many diverse clinical situations including: glycaemic control and sepsis diagnosis in an intensive care setting [19], monitoring the impact of type 2 diabetes therapy [20], and monitoring the impact of drug therapy [21]. Clinical studies also investigated $S I$ as a metabolic health marker [22, 23]. Finally, such a knowledge of $S I$ could aid glycaemic regulation and the development of personalised treatment plans for newly diagnosed diabetic individuals.

The dynamic insulin sensitivity test (DIST) is a recently developed low-dose, short-protocol test similar to the IMIVGTT [17, 24]. The subject's response to a relatively low dose (5-20g) IV glucose bolus followed by a low dose (0.5$2 \mathrm{U}) \mathrm{IV}$ insulin bolus is monitored with samples assayed for glucose, insulin and C-peptide. These concentrations are used to identify model-based parameters for $S I$, endogenous insulin secretion $\left(U_{e n}\right)$ and liver clearance $\left(n_{L}\right)$ by solving the physiological model shown in Fig. (1). The model is a compartmental pharmaco-kinetic (PK) and pharmacodynamic (PD) model representing the physiological pathways and interactions between insulin, C-peptide and glucose in the human body [25]. Prior modelling studies have shown the $S I$ value from the model can be highly correlated to the insulin sensitivity metric (ISI) from the EIC $(\mathrm{R}=0.99)$ [17], and a clinical validation study is ongoing with initial correlations of $R \approx 0.78$. Note that the DIST differs from the IM-IVGTT by measuring every 5-10 minutes and using significantly lower insulin and glucose doses.

This research presents and evaluates the quick DIST method (DISTq) which incorporates a novel method, assumptions and mathematical process to identify SI. The method only requires the glucose measurements from the previously presented DIST protocol $[17,24]$, and anatomical and demographic data available at the time of testing (height, weight, sex and age). Hence, relatively costly insulin and Cpeptide samples that take 1-3 days to process in a separate lab are not needed and effectively real-time estimation is enabled. The method uses the DIST protocol and populationbased parameter values to estimate the subject's insulin concentrations during testing to replace these missing assays. This ability to estimate insulin concentrations in the subject is unique to the DISTq and in direct contrast to other surrogate $S I$ tests that do not measure (or estimate) insulin and are therefore poorer predictors of $S I$ and its changes due to an intervention.

\section{METHOD}

The DISTq solver method is based on model parameter and concentration estimates in an individual. These estimates are functions of the relationship between $S I$ and various

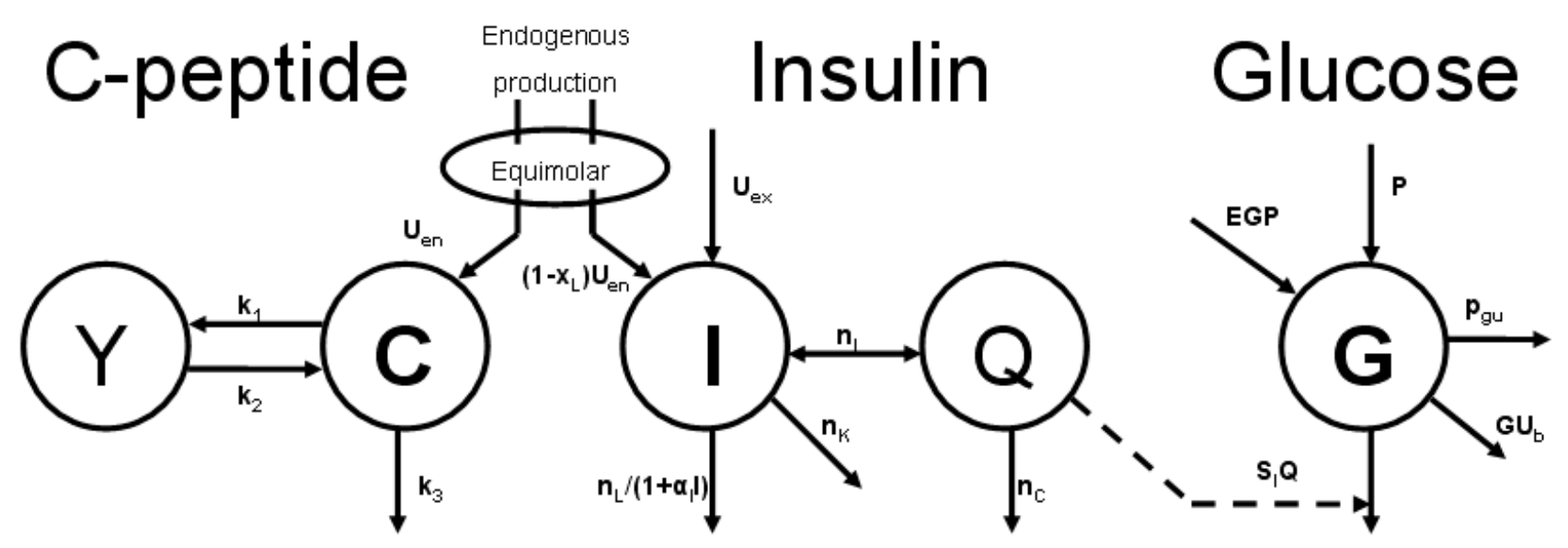

Fig. (1). The physiological compartmental model used to match the DIST test data (symbols are fully defined in the Methods section). 
anatomical and/or physiological factors, and are used to approximate the subject's insulin concentrations in the absence of direct insulin and C-peptide measurements. The DISTq is thus a subset of the fully sampled DIST $[17,24]$.

\subsection{DIST Model}

The DISTq method utilises the model developed in the pilot study of the fully sampled DIST test [17, 24]. The model is shown in Fig. (1) and is mathematically expressed:

\section{C-Peptide Pharmaco-Kinetics}

$\dot{C}=-\left(k_{1}+k_{3}\right) C+k_{2} Y+\frac{U_{e n}}{V p}$

$\dot{Y}=k_{1} C-k_{2} Y$

\section{Insulin Pharmaco-Kinetics}

$\dot{I}=-n_{K} I-n_{L} \frac{I}{1+\alpha_{I} I}-\frac{n_{I}}{V p}(I-Q)+\frac{U_{e x}}{V p}+\left(1-x_{L}\right) \frac{U_{e n}}{V p}$

$\dot{Q}=\frac{n_{I}}{V q} I-\left(n_{C}+\frac{n_{I}}{V q}\right) Q$

\section{Glucose-Insulin Pharmaco-Dynamics}

$$
\dot{G}=-p_{g u}\left(G-G_{e}\right)-S I\left(G Q-G_{e} Q_{b}\right)+\frac{P}{V g}
$$

where: $k_{1}, k_{2}, k_{3}, n_{K}, n_{L}$, and $n_{C}$ are rate parameters $\left[\mathrm{min}^{-1}\right] ; n_{I}$ is the transport rate between plasma and interstitium $\left[\mathrm{L} \cdot \mathrm{min}^{-1}\right]$; $\alpha_{I}$ is the saturation coefficient of liver clearance $\left[\mathrm{L} \cdot \mathrm{mU}^{-1}\right] ; C$ and $Y$ are plasma and interstitial compartment C-peptide concentrations $\left[\mathrm{pmol} \cdot \mathrm{L}^{-1}\right] ; U_{e n}$ is the rate of endogenous insulin and (equimolar) C-peptide production $\left[\mathrm{mU} \cdot \mathrm{min}^{-1}\right] ; I$ and $Q$ are plasma and interstitial compartment insulin concentrations $\left[\mathrm{mU} \cdot \mathrm{L}^{-1}\right] ; U_{e x}$ and $P$ are the insulin and glucose bolus inputs [mU and $\mathrm{mmol}$; $V p$ and $V q$ are volumes of distribution of plasma and interstitium, respectively $[\mathrm{L}] ; x_{L}$ is the fractional first pass liver extraction $\left[\mathrm{mU} \cdot \mathrm{mU}^{-1}\right] ; G$ is the glucose concentration in plasma $\left[\mathrm{mmol} \cdot \mathrm{L}^{-1}\right] ; G_{e}$ and $Q_{b}$ are equilibrium or basal levels of the respective analytes $\left[\mathrm{mmol} \cdot \mathrm{L}^{-1}\right.$ and $\left.\mathrm{mU} \cdot \mathrm{L}^{-1}\right] ; V g$ is the volume of distribution of glucose [L]; and $p_{g u}$ is the non-insulin mediated glucose disposal rate $\left[\mathrm{min}^{-1}\right]$.

\subsection{DIST Pilot Protocol}

Sixteen subjects were recruited and underwent a total of 46 DIST tests in the DIST pilot study [24]. Subjects reported to the place of testing in the morning, having fasted overnight. All tests were completed in a seated position with a cannula inserted into the antecubital fossa, through which glucose (50\% dextrose) and insulin (actrapid) boluses were administered and blood samples taken. Subjects had blood samples taken at $0,10,15,20,25,30,35,40,50,60$ minutes. The glucose and insulin injections immediately followed the 10 and 20 minute samples, respectively. Timing of samples and boluses were kept to the closest minute. Three dosing protocols were used in this pilot study:

- Low - $5 \mathrm{~g}$ glucose, $0.5 \mathrm{U}$ insulin
- Medium $-10 \mathrm{~g}$ glucose, $1 \mathrm{U}$ insulin

- High $-20 \mathrm{~g}$ glucose, $2 \mathrm{U}$ insulin.

All samples were assayed for glucose (Enzymatic glucose hexokinase assay, Abbot Labs, Illinois USA), insulin and C-peptide (ELISA Immunoassay, Roche, Mannheim, Germany). Further details of the participant attributes, protocol and results can be found in [24].

\subsection{Requirements for DISTq Profile Estimation}

The DISTq method was developed to provide an accurate estimation of $S I$ in clinical real-time, meaning a diagnostic outcome can be generated within 5 minutes of test completion. Insulin and C-peptide assays need to be performed in a laboratory and thus do not provide immediate results. However, precise estimation of $S I$ is not possible without an accurate indication of insulin concentrations.

DISTq overcomes this lack of information by utilising population-based assumptions to estimate insulin concentrations in an individual. Fig. (2) shows a typical subject-specific simulated response to the DIST protocol. Shown are endogenous insulin secretion rate $U_{e n}(t)$, and plasma and interstitial insulin concentrations, $I(t)$ and $Q(t)$. The qualitative shape of the kinetics shown are relatively consistent between subjects. However, the magnitude of the kinetics varies with dosing and (diagnosed) diabetic or metabolic status.

Comprehensive and unique endogenous insulin production and insulin concentration profiles can be simulated with knowledge of the 10 kinetic features shown in Fig. (2). Five of these 10 characteristics can be isolated by the protocol, or the physiological assumptions used in the full DIST test. These five characteristics are listed below with numbering in accordance to the corresponding features shown in Fig. (2) and an explanation of how they are determined:

2. The first phase $U_{e n}$ response (initial secretion peak) is assumed to begin immediately or within 1 minute of the glucose bolus with known timing [26].

6. The first phase $U_{e n}$ will be visibly manifested in $I(t)$.

7. The administration time of the insulin bolus is recorded during the trial.

8. The concentration of insulin in plasma immediately following the insulin bolus is readily defined by the mass of the bolus divided by the plasma distribution volume $(V p)$.

10. The rate of insulin diffusion and transport between plasma and interstitial compartments has been defined by [27]. Thus, $Q(t)$ can be defined solely as a function of transport rates, a steady state concentration ratio $(\gamma)$, and the measured or calculated $I(t)$ profile.

The five remaining kinetic features shown in Fig. (2) cannot be readily inferred by the structure of the protocol, the model used, or the specific measurements, and must be derived using other methods. These features are listed below with numbering in accordance to Fig. (2):

1. The basal $U_{e n}$ production rate $\left(U_{b}\right)$ 

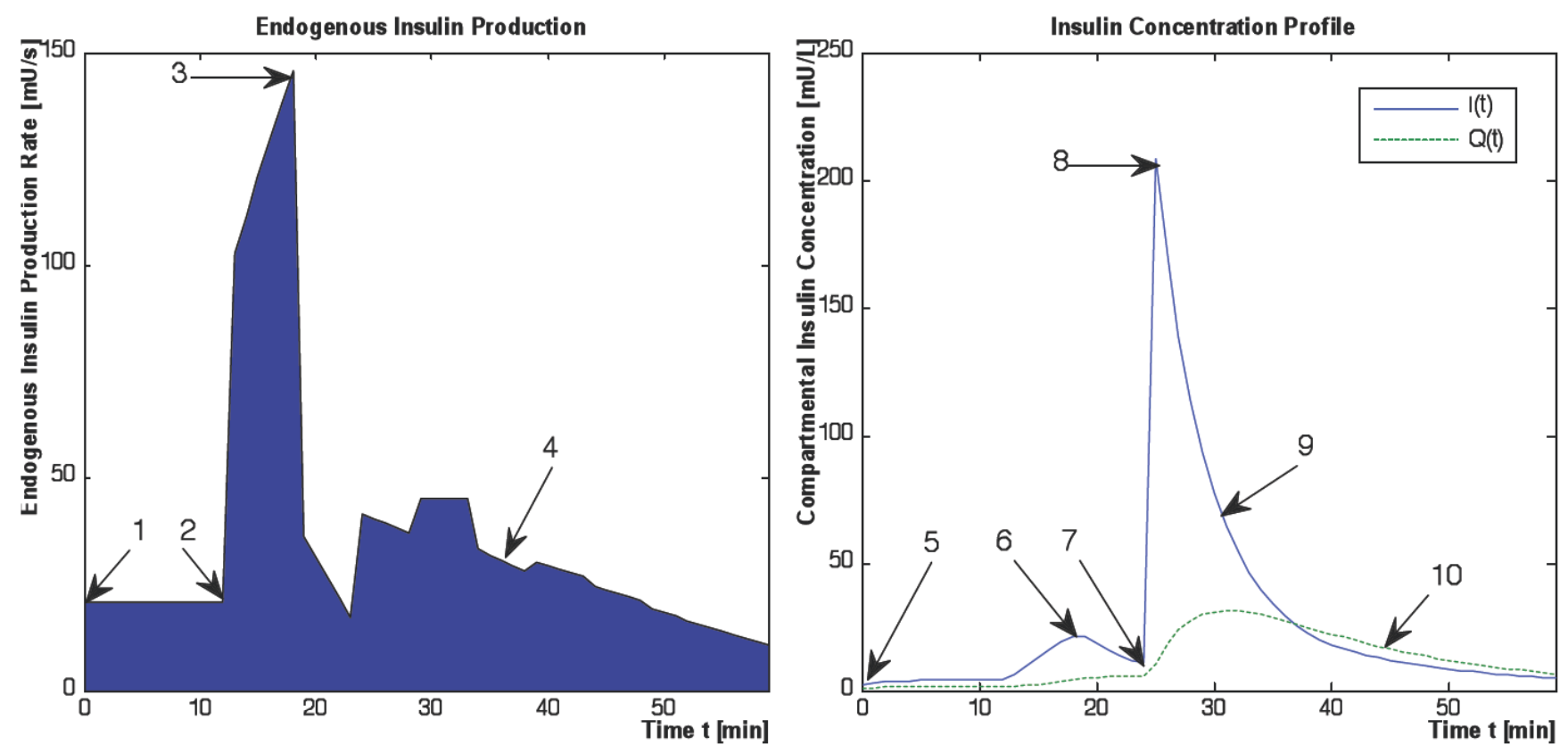

Fig. (2). A typical endogenous insulin production and resulting insulin concentration response to the DIST protocol. Shown are the endogenous insulin secretion rate profile $U_{e n}(t)$ (left), and the plasma and interstial insulin concentrations, $I(t)$, $Q(t)$, (right). The kinetic features required to describe the profiles in the absence of insulin and C-peptide data are shown by numbers 1-10.

3. The maximal first phase response to the glucose bolus $\left(U_{\max }\right)$

4. The degree to which the second phase response will be suppressed by elevated insulin concentrations after the exogenous insulin bolus $\left(U_{\text {ave }}\right)$

5. The basal insulin concentration in plasma $\left(I_{b}\right)$

9. The rate of liver disposal of insulin from plasma $\left(n_{L}\right)$

Mathematical or a-priori relationships between these parameters are not evident or reported elsewhere. However, relationships were apparent between these parameters and $S I$ derived from the full test data. After the parameters of interest and SI were solved using data from the fully sampled DIST tests, the parameters were plotted against SI and trends became apparent. These trends can be seen in Fig. (3). The DISTq method utilises these trends to enable a-posteriori parameter estimation and unique simulations of endogenous and overall insulin kinetics responses to the test protocol.

\subsection{Full DIST Test Data Analysis}

The results from the fully sampled DIST tests are used to develop the population equations characterising the trends in insulin kinetic parameters. Equations (1) to (5) are the pharmacokinetic, multi-compartmental equations used to derive the model parameters from the DIST data $[17,24]$.

Equations (1) and (2) can be solved directly to find $U_{e n}(\mathrm{t})$ using a linear interpolation (evaluated at 1 minute intervals) of the C-peptide data $C(t)$, and a Picard iteration to find $Y(t)$. A Picard iteration is capable of solving non-linear systems using discretised transforms of the governing ODEs [28, 29]. In this case, the iteration uses updating predictions of the $Y(t)$ profile to converge to a highly accurate $Y(t)$.

$\dot{Y}(t)=k_{1} C(t)-k_{2} Y(t) \stackrel{\text { therefore }}{\longrightarrow} \int \dot{Y}(t) d t=k_{1} \int C(t) d t-k_{2} \int Y(t) d t$
$Y(t)=Y_{0}+k_{1} \int_{0}^{t} C(t) d t-k_{2} \int_{0}^{t} Y(t) d t$

Therefore, $U_{e n}(t)$ can be obtained directly using Equation (7) for $\mathrm{Y}(\mathrm{t})$ and C-peptide assay data, as defined:

$U_{e n}(t)=\dot{C}(t)+\left(k_{1}+k_{3}\right) C(t)-k_{2} Y(t) V_{p}$

where $\dot{C}(t)$ is evaluated plasma C-peptide concentration change at one minute intervals using the interpolated minutewise $C(t)$ profile.

The insulin data can be used to obtain a value for the liver clearance parameter $\left(n_{L}\right)$. To identify $n_{L}$, an integral formulation of Equations (3) and (4) similar to [30] is used. The first step is to define $Q(t)$. The iterative integral method is sufficiently robust that interpolated initial guesses allow later iterations to produce highly accurate re-simulations of insulin concentration. Thus, the initial $I(t)$ estimate is simply a linear interpolation of the measured insulin data and the initial $Q(t)$ is equal to half $I(t)$ as defined by the steady state ratio of insulin concentration between the plasma and interstitium $(\gamma)$ :

$\frac{Q_{s s}}{I_{s s}}=\gamma=\frac{Q(t)_{\mathrm{int}}}{I(t)_{\mathrm{int}}}$

Integrating Equation (3) from 0 to $t$ for the given estimate $Q_{\text {int }}(t)$ of Equation (9), and assuming $I(t)$ is a linear interpolation of the measured data yields:

$$
\begin{aligned}
& -n_{L} \int_{0}^{i} \frac{I}{1+\alpha_{I} I} d t=I_{\text {meas }}(t)-I_{b}+\left(n_{K}+\frac{n_{I}}{V p}\right) \int_{0}^{i} I d t \\
& -\frac{n_{I}}{V p} \int_{0}^{i} Q_{\text {int }} d t-\int_{0}^{i} \frac{\left(U_{e x}+\left(1-x_{L}\right) U_{e n}\right)}{V p} d t
\end{aligned}
$$

where: $\mathrm{I}_{\mathrm{b}}=\mathrm{I}_{\text {meas }}(0)$ and $\mathrm{I}=\mathrm{I}_{\text {int }}(\mathrm{t})$. 
Choosing $\mathrm{N}$ time points in Equation (10), corresponding to the number of data points, gives $\mathrm{N}$ equations with one unknown, $n_{L}$. These equations can be readily solved by linear least squares to give an initial estimate for the value of $n_{L}$. For this given value of $n_{L}$, Equations (3) and (4) are then numerically solved using a Picard iteration [28, 29] to determine a new $I(t)$ and $Q(t)\left(I_{\text {new }}(t)\right.$ and $\left.Q_{\text {new }}(t)\right)$. Depending on the accuracy of the first estimate, $I(t)$ and $Q(t)$ may not yet give an optimal match to the data. The reason for this error is that the initial linear interpolation to the measured $I(t)$ may not capture the true peaks, introducing error into the integral terms of Equation (10). A solution to this problem is to replace $I_{\text {int }}(t)$ and $Q_{\text {int }}(t)$ in Equation (10) with $I_{\text {new }}(t)$ and $Q_{\text {new }}(t)$, then determine a new $n_{L}$ by a similar linear least square approach. This process can be continued until convergence is reached. In contrast to the methods presented for the fully sampled DIST test pilot study [24] the first pass extraction parameter $x_{L}$ is fixed at a population average value of 0.7 [31-33].

A similar method is applied to the glucose model, Equation (5), with two variable parameters to be identified, $S I$ and $V g$. Integrating Equation (5) from 0 to t yields:

$$
\begin{aligned}
& G_{\text {meas }}(t)-G_{0}=-p_{g u} \int_{0}^{t}\left(G_{\text {int }}(t)-G_{e}\right) d t-S I \int_{0}^{t}\left(G_{\text {int }}(t) Q(t)-G_{e} Q_{b}\right) d t+ \\
& \frac{1}{V g} \int_{0}^{t} P(t) d t
\end{aligned}
$$

Choosing $\mathrm{N}$ values of time $\mathrm{t}=\mathrm{t}_{1}, \ldots, \mathrm{t}_{\mathrm{N}}$ in Equation (11) gives a matrix system defined:

$$
A \bullet\left[\begin{array}{c}
S I \\
1 / V g
\end{array}\right]=b
$$

where:

$$
\begin{gathered}
A=\left[\begin{array}{cc}
\int_{0}^{t_{1}}\left(G_{\mathrm{int}} Q-G_{e} Q_{b}\right) d t & \int_{0}^{t_{1}} P d t \\
\int_{0}^{t_{2}}\left(G_{\mathrm{int}} Q-G_{e} Q_{b}\right) d t & \int_{0}^{t_{2}} P d t \\
\vdots & \vdots \\
\int_{0}^{t_{N}}\left(G_{\mathrm{int}} Q-G_{e} Q_{b}\right) d t & \int_{0}^{t_{N}} P d t
\end{array}\right] \text { and } \\
b=\left[\begin{array}{c}
G_{\text {meas }}\left(t_{1}\right)-G_{0}+p_{g u} \int_{0}^{t_{1}}\left(G_{\mathrm{int}}-G_{e}\right) d t \\
G_{\text {meas }}\left(t_{2}\right)-G_{0}+p_{g u} \int_{0}^{t_{2}}\left(G_{\mathrm{int}}-G_{e}\right) d t \\
\vdots \\
G_{\text {meas }}\left(t_{N}\right)-G_{0}+p_{g u} \int_{0}^{t_{N}}\left(G_{\mathrm{int}}-G_{e}\right) d t
\end{array}\right]
\end{gathered}
$$

Solving Equation (12) by linear least squares provides an initial estimate to $S I$ and $V g$, as was done with $n_{L}$ in Equation (10). $G_{\text {int }}(t)$ is re-simulated using the estimated values for $S I$ and $V g$ and substituted into the integrals of Equation (12) to determine a new matrix $A$ and vector $b$ in Equation (13).
Equation (12) is solved again by linear least squares and this process is continued until convergence.

The DISTq process utilises parameter estimations to predict the insulin concentration profile of a subject during a DIST test. Using the methods described above, the parameters required for this process have been defined for the full DIST data set. The goal is to estimate these parameters based on population relationships between their values resulting from the DIST as defined above. The paramters to be identified are:

- $U_{b}$ : the first value of $U_{e n}(t)$ - (deconvolution of Equation (8))

- $U_{\max }$ : the maximum value of $U_{e n}(t)$ - (deconvolution of Equation (8))

- $U_{\text {ave }}$ : the average value of $U_{e n}(t)$ - (deconvolution of Equation (8))

- $I_{b}$ : the basal insulin measurement - (direct measurement)

- $n_{L}$ : the liver clearance rate - (identification of Equation (10))

- SI: insulin sensitivity - (identification of Equation (12))

\subsection{Population Based Parameter Estimation}

The population-based parameters $\left(U_{b}, U_{\max }, U_{a v e}, I_{b}\right.$ and $\left.n_{L}\right)$ are compared to $S I$ from the full DIST $[17,24]$ and anatomical parameters (height, weight, BMI, BSA) obtained from the individuals. The population based parameters show a stronger relationship to $S I$ in Fig. (3) than to any a-priori patient-specific information that would be available at the time of testing. In particular, $U_{b}, U_{a v e}$ and $I_{b}$ all have a power relationship with SI. $U_{\max }$ does not have a strong relationship to $S I$, but is also estimated with a power relationship. In contrast, $n_{L}$ is predicted using a $\log$ relationship to $S I$. Equations (14) to (18) define the mathematical relationships between the parameters (shown as the blue lines in Fig. 3). Note that no dispensation has been made to adjust any parameters for different dosing, which adds to some of the spread seen in Fig. (3), and thus providing a conservative trend.

$$
\begin{aligned}
& U_{b}=122.49 *(S I)^{-0.7236} \\
& U_{\text {max }}=168.32 *(S I)^{-0.1821} \\
& U_{\text {ave }}=177.87 *(S I)^{-0.6932} \\
& I_{b}=63.18 *(S I)^{-1.1347} \\
& n_{L}=0.0491 * \ln (S I)+0.0447
\end{aligned}
$$

\subsection{DISTq Method}

DISTq is an iterative method that uses an initial population average $S I$ value $\left(S I=10^{-4} \mathrm{~L} \cdot \mathrm{min}^{-1} \cdot m U^{-1}\right)$ in Equations (14) to (18) to provide an initial estimate for the unknown parameters needed to generate an interstitial insulin profile $Q(t)$. This $Q(t)$ profile can then be used with just the glucose data and Equation (12) to generate a new, 


\section{Trends Between Insulin Sensitivity and the Population-Based Parameters}
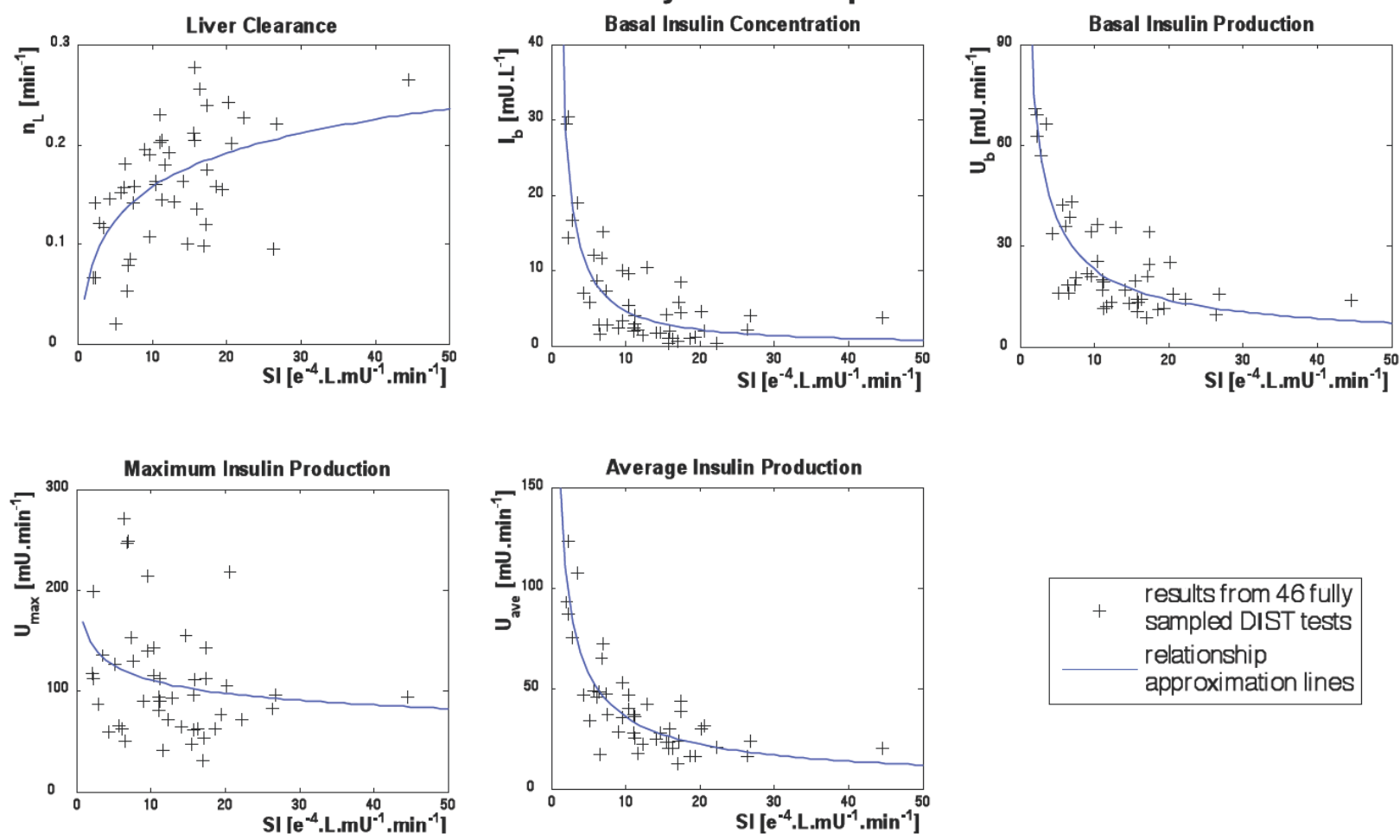

Fig. (3). Trends between insulin sensitivity and the five population-based parameters that cannot be defined with a-priori identification methods. The blue lines are representative of the approximation equations that are used to identify the parameters (Equations 14-18).

more accurate prediction of $S I$. This new $S I$ is then used in Equations (14) to (18) to redefine the unknown parameters: $U_{b}, U_{\text {max }}, U_{\text {ave }}, I_{b}$ and $n_{L}$. In turn, these redefined parameter values are used to re-simulate a new $Q(t)$ profile. Equation (12) can then be solved again with the glucose data for a new, updated prediction of $S I$. This process is iterated up to five times or until SI stabilises to within $0.01 \%$ change between iterations. Fig. (4) shows this process schematically, denoted as the first iterative loop in the process.

A second, subsequent iterative cycle can be used to further increase the accuracy of the derived SI. If the glucose data following the insulin bolus is of sufficient resolution, an inference can be made into the shape of the insulin decay curve. Once the initial iterative process has converged to a value for $S I$, the value of $n_{L}$ is allowed to vary up to $\pm 20 \%$ and $S I$ is then further refined. The $n_{L}$ value that minimises the least-square error in the re-simulated glucose concentrations is used to define the new (second iteration) value for SI. This second cycle is also shown in Fig. (4).

\subsection{Analyses}

\subsubsection{Part one: Clinical Equivalence of the DIST and DISTq Solver Methods}

It is important to determine whether accurate $S I$ estimation is possible when insulin and C-peptide data are not used. To assess this, $S I_{D I S T q}$ is compared to the fully sampled $S I_{D I S T}$ using a Pearson correlation coefficient. Results for both the basic DISTq method ( $1^{\text {st }}$ iteration) and the additional $n_{L}$ variation ( $2^{\text {nd }}$ iteration) are compared to the full DIST test value.
Furthermore, the DISTq method's dependence on their initial $S I$ estimations is evaluated by comparing estimated $S I_{D I S T q}$ values obtained using initial $S I$ estimations from differing ends of the expected range $\left(2\right.$ to $\left.30 \mathrm{~L} \cdot \mathrm{mU}^{-1} \cdot \mathrm{min}^{-1}\right)$. If the DISTq method is independent of the initial SI estimate, the resulting $S I_{D I S T q}$ values for any trial data should be identical.

\subsubsection{Part Two: Separating the Derivation Set from the Validation Set}

If the DISTq is to be used exclusively in a clinical study, the population characteristics will not necessarily be known. To address this issue, the validation data set is isolated from the derivation data set. In particular, the parameter relationships of Equations (14) to (18) are generated from the results of 37 (of 46) randomly-selected full DIST trials. The remaining 9 trials are used as an independent validation set to compare the DISTq result to the fully sampled DIST results. This random selection and analysis is repeated 25 times. The derived $S I$ values from the 25 validation sets are then correlated to the full DIST SI results for the matching test sets to assess any significant loss of correlation when the randomly selected derivation set is isolated from the validation set.

\subsubsection{Part three: Monte Carlo Analysis of the DISTq Method}

Finally, a comparison can be made between the coefficient of variation $(\mathrm{CV}=$ standard deviation divided by the mean $S I$ ) of $S I_{D I S T q}$ and the fully sampled $S I_{D I S T}$, to obtain an indication of the expected robustness and error of the DISTq relative to the DIST and the euglycaemic clamp as 


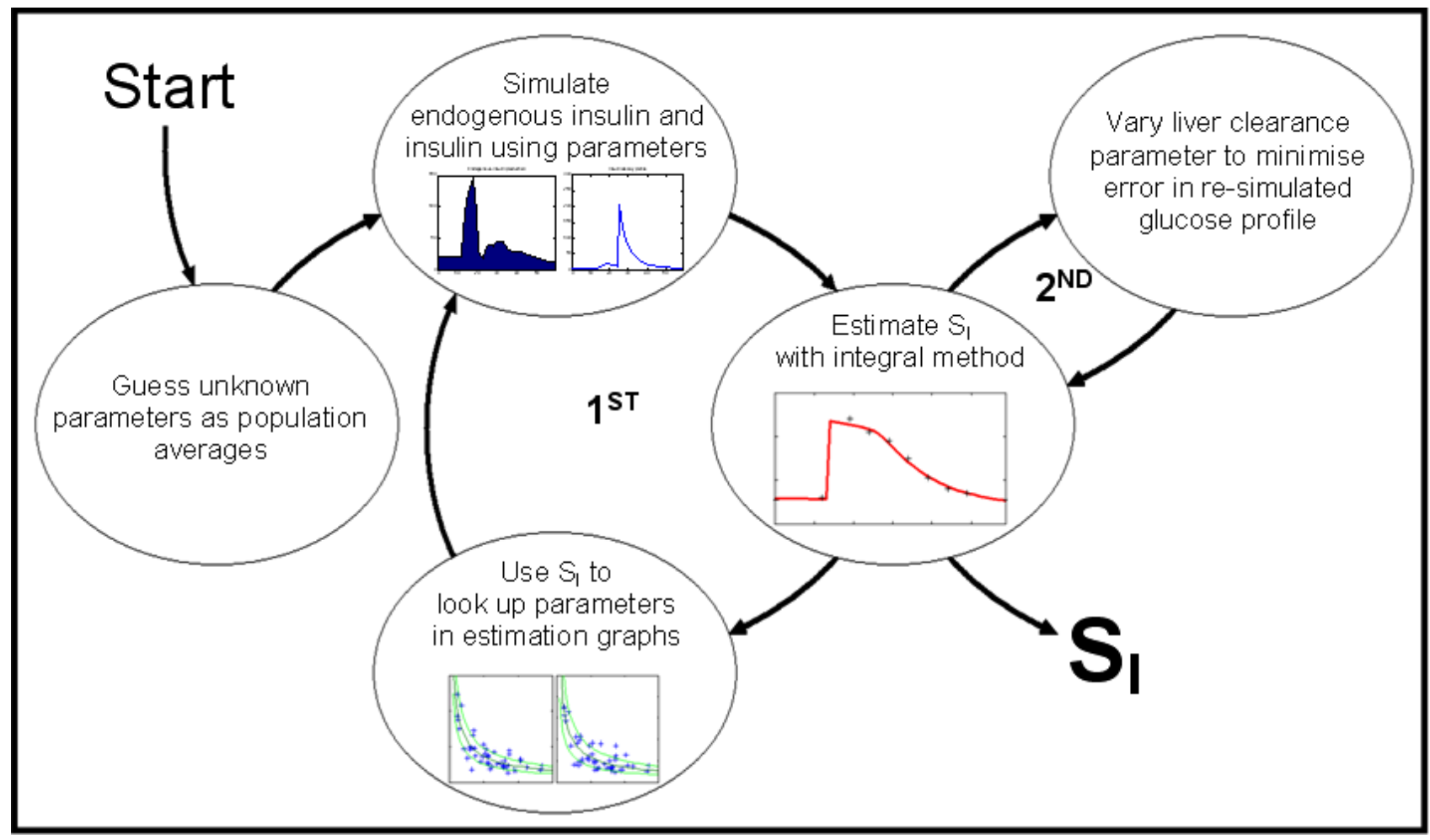

Fig. (4). A schematic of the DISTq iterative solver cycles (Including optional second cycle to optimise liver clearance rate). The first cycle is iterated five times to identify $S I$. The second cycle can be used to further refine the resultant $S I$ by varying the $n_{L}$ parameter.

reported in [17]. A Monte-Carlo analysis of the fully sampled DIST has been previously completed [17], and the procedure of this study is repeated for both the fully sampled DIST and DISTq.

The data used to generate simulation results was taken from 146 euglycaemic clamp tests performed during a lifestyle intervention study on 73 individuals [20]. Virtual Cpeptide, insulin and glucose data for a DIST test can be simulated using the physiological model defined by Equations (1) to (5) and an SI value identified from the euglycaemic clamp test results [17]. Virtual discrete "samples" are taken per the DIST protocol definition by isolating the derived concentrations at the appropriate time points. The Monte Carlo simulation adds noise to these virtual samples in accordance with published assay and dosing errors. Further details of the simulation protocol can be found in [17]. Finally, differing random levels of endogenous glucose suppression are also included. Each test is run with random errors 250 times for each trial creating 36500 analyses, which are use to calculate the CV.

The computationally expensive added $n_{L}$ variation method was not applied to minimise computational time. Hence, the results will be conservative in the estimation of the overall DISTq error, without added refinement.

\section{RESULTS}

\subsection{Part one: Clinical Equivalence of the DIST and DISTq Methods}

The basic DISTq method showed a strong correlation to the fully sampled DIST test $S I(\mathrm{R}=0.86)$, which improved to $\mathrm{R}=0.91$ using the $n_{L}$ parameter variation method. Fig. (5) shows the relationship between the $S I_{D I S T}$ solved with the full data set and the iterative-integral method, and the $S I_{D I S T q}$ using limited data and the DISTq method.

The correlation between DISTq SI values obtained using initial $S I$ estimates from distant ends of the expected range was perfect $(\mathrm{R}=1.00)$, showing that the SI values generated with the DISTq method are independent of the initial SI estimation. Thus, the DISTq method is convex and robust with respect to SI starting value, as it generated identical results, regardless of starting point.

\subsection{Part two: Separating the Derivation Set from the Validation Set}

Fig. (6) shows the relationship between the fully sampled $S I_{D I S T}$ and $S I_{D I S T q}$ when the validation test set is isolated from the set used to derive Equations (14) to (18). Although there is a marginal decrease in the correlation from Fig. (5), a strong correlation is maintained $(\mathrm{R}=0.83$ and $\mathrm{R}=0.88$ for the fixed and variable $n_{L}$ methods respectively). As the simulation method randomly selected different sub-sets of tests to generate population parameters and to validate these, some tests were selected more frequently in the parameter generation than others. However, each trial was part of the validation set at least once.

For those data sets in the validation set several times, the vertical clusters of $S I_{D I S T q}$ in Fig. (6) show the DISTq variability at different levels of $S I_{D I S T q}$ induced by the differences in development data set. Fig. (6) shows that most such results are robust, especially at the clinically more important lower $S I_{D I S T}$ levels. However, it is also clear that a larger derivation data set would provide a potentially more 


\section{Comparision Between SI Metrics from the DIST and DISTq Methods}
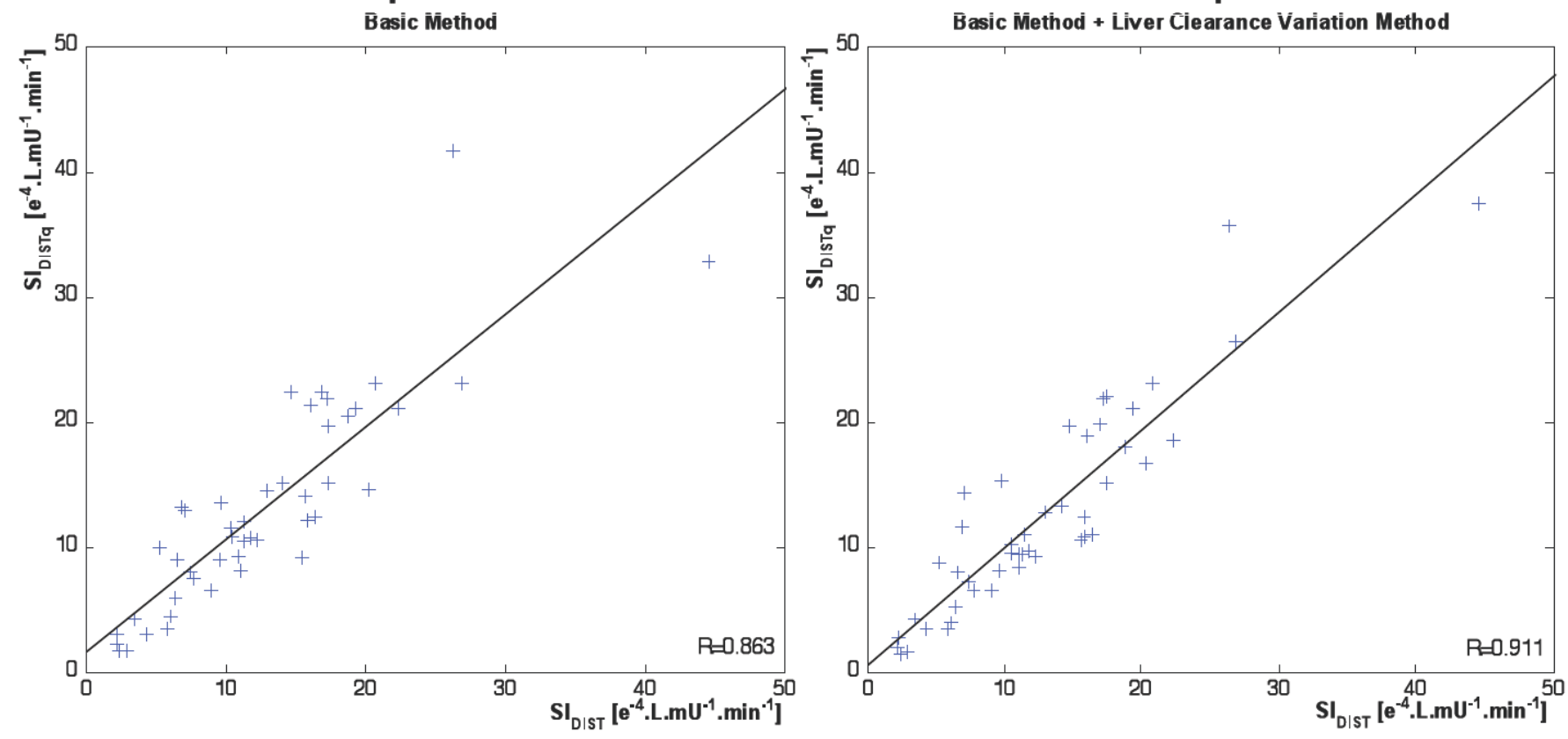

Fig. (5). Relationships between $S I_{D I S T}$ from the fully sampled DIST tests and $S I_{D I S T q}$ for the basic DISTq method (left), and with the additional variation of liver clearance cycle (right).

\section{Comparison Between SI from the DIST and DISTq Methods with Isolated Derivation Data}
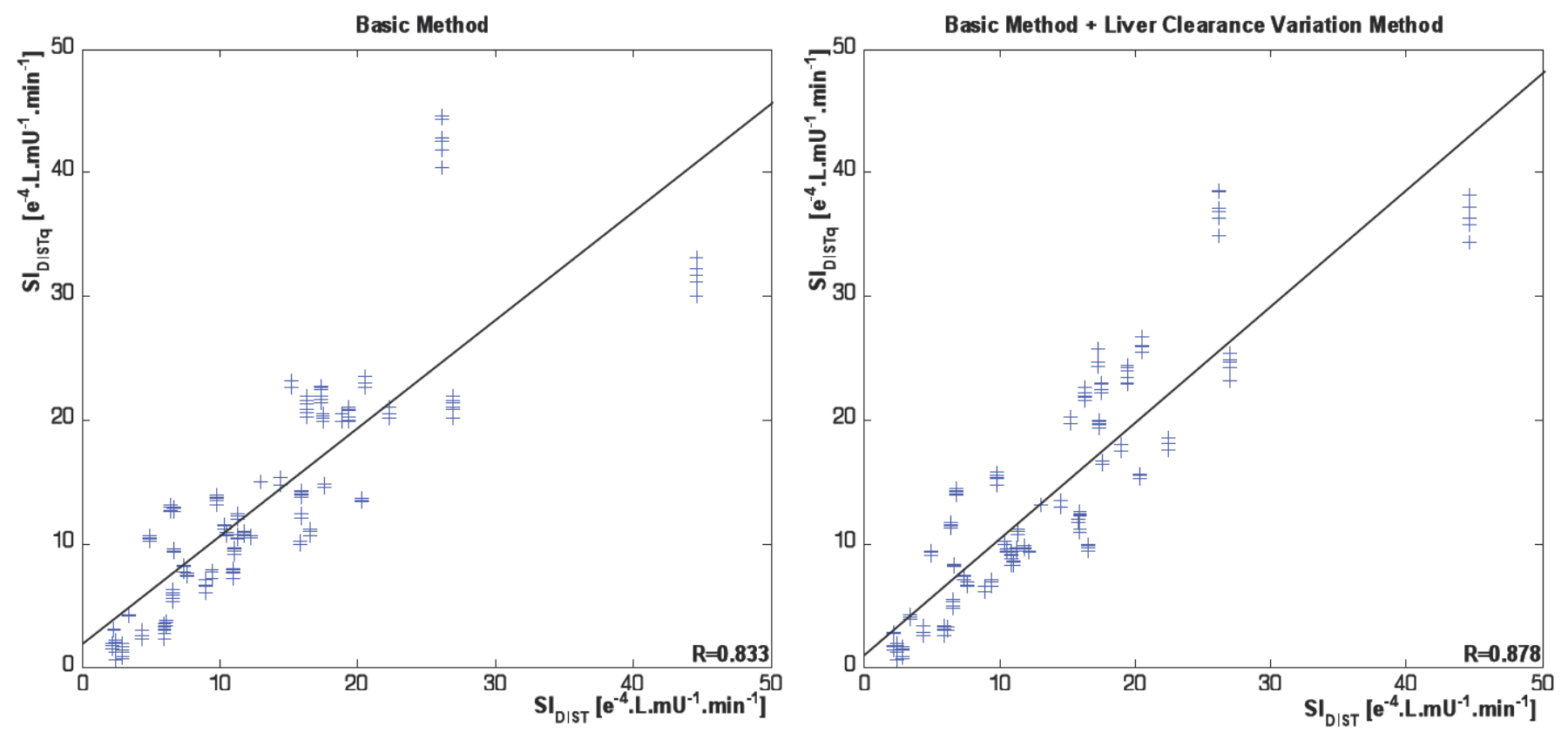

Fig. (6). A comparison between the fully sampled $S I_{D I S T}$ and the $S I_{D I S T q}$ from the DISTq method when the population-based equations (Equations 14-18) are derived using data from a subset of trials which are isolated from the validation set $(\mathrm{N}=225$; 25 iterations with a randomly selected 37 trial equation derivation set, and 9 solved trials per iteration).

robust test. Overall, Fig. (6) does show that the estimates of Equations (14) to (18) are generally stable and effective.

\subsection{Part Three: Monte Carlo Analysis of the DISTq Method}

The Monte Carlo analysis of the fully sampled DIST test confirmed the results of the initial investigation [17]. These simulations showed a strong correlation between $S I_{D I S T}$ and the sensitivity metric of the euglycaemic clamp (ISI) $(\mathrm{R}=0.92)$. The correlation is improved when ISI is normalised by the steady state glycaemic concentration achieved during the euglycaemic clamp ( $\left.\operatorname{ISI}_{\mathrm{G}}\right)(\mathrm{R}=0.98)$. This result is very similar to the findings in [17], which found $\mathrm{R}=0.93$ and $\mathrm{R}=0.99$ respectively. 

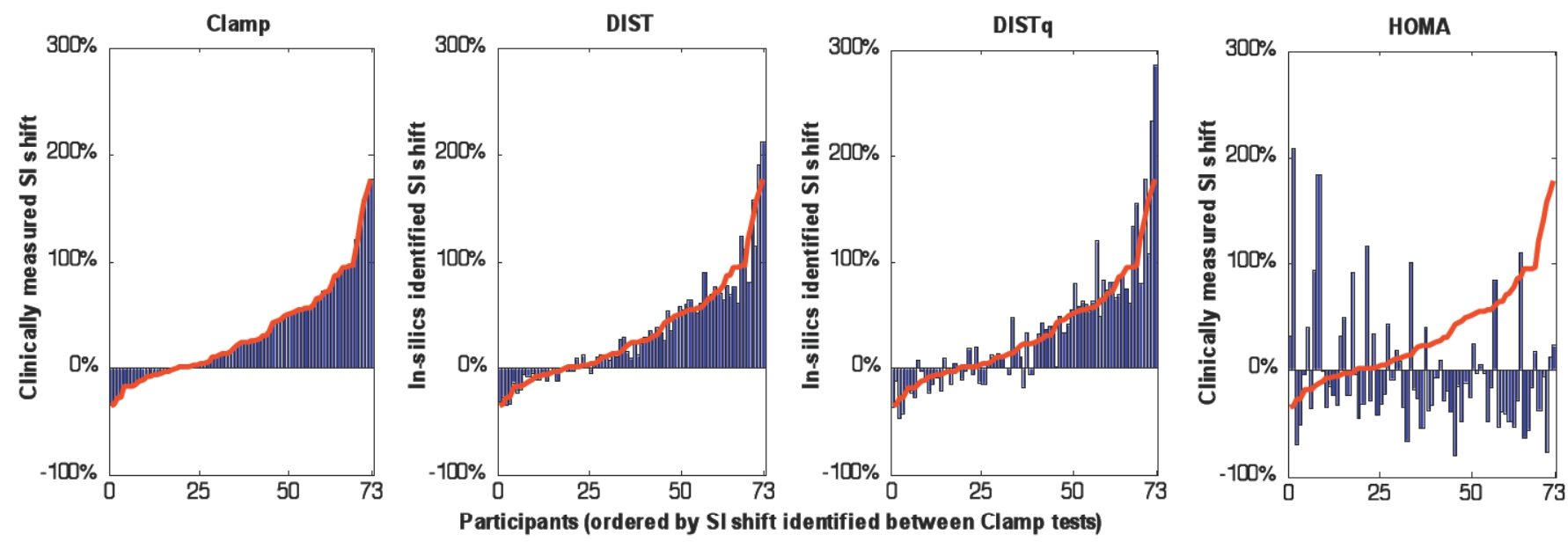

Fig. (7). The SI shift measured in-silico by the DIST and DISTq methods compared to the clinically measured euglycaemic clamp and HOMA derived SI shift. The SI values are sorted increasing from left to right by the $S I$ shift observed between euglycaemic clamp tests. The red line shows the clamp derived $S I$ shift overlaid on the results from the other tests.

The virtual $S I_{D I S T q}$ correlated well to ISI ( $\left.\mathrm{R}=0.81\right)$, which again improved when compared to $\mathrm{ISI}_{\mathrm{G}}(\mathrm{R}=0.89)$. The HOMA (homeostatic model assessment) [18] correlation was much poorer when compared to ISI and $\mathrm{ISI}_{\mathrm{G}}$ (ISI: $\mathrm{R}=-0.37$ and $I_{S I}$ : R=-0.37). $S I_{D I S T q}$ correlated better to $S I_{D I S T}$ in this in-silico analysis than the clinical results $(\mathrm{R}=0.96$ compared to $\mathrm{R}=0.83-0.91$ ).

The potentially most important component of an $S I$ test is the ability to define changes in SI accurately over time, to monitor intervention or treatment. As expected, the DIST test showed a stronger ability to capture the insulin sensitivity shift seen in the euglycaemic clamp tests $(\mathrm{R}=0.97)$ than the DISTq $(\mathrm{R}=0.92)$. Both DIST methods performed significantly better than HOMA $(\mathrm{R}=-0.22)$. Fig. (7) shows the $S I$ shift over four tests, sorted by increasing $S I$ shift according to the clinically measured change in euglycaemic clamp results for the 73 subjects of the lifestyle intervention trial used in the Monte Carlo analysis [20]. It is visually clear that the DIST and DISTq both capture the clinically relevant changes, and that the HOMA metric is not clinically effective for tracking these changes due to intervention or any equivalently modest but clinically significant changes over time.

This Monte Carlo simulation found a slightly higher average $\mathrm{CV}$ for the fully sampled DIST $(\mathrm{CV}=6.6 \%)$ than the value found in [17] $(\mathrm{CV}=4.5 \%)$. The average $\mathrm{CV}$ for DISTq

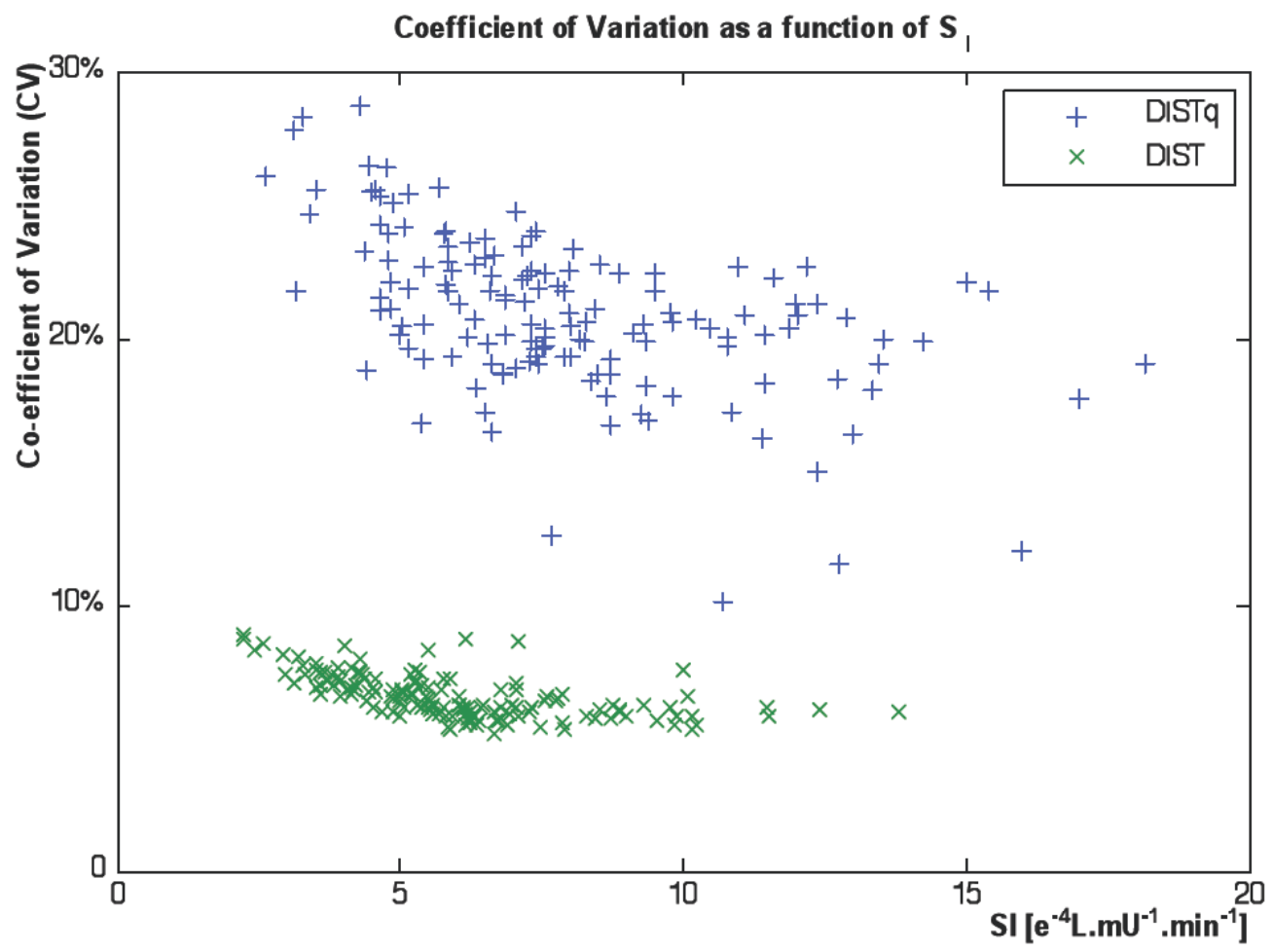

Fig. (8). Coefficients of variation from the Monte Carlo analysis of the DIST and DISTq methods. 
was significantly higher $(\mathrm{CV}=21.2 \%)$. Fig. $(8)$ shows that the $\mathrm{CV}$ was higher for the more insulin resistant subjects, toward a maximum of $30 \%$ for the most insulin resistant subject. The average HOMA CV was significantly lower than the DISTq $(\mathrm{CV}=10 \%)$ but does not capture changes in $S I$ well as seen in Fig. (7).

\section{DISCUSSION}

\subsection{Correlations and Potential Limitations}

A strong correlation between $S I_{D I S T}$ and $S I_{D I S T q}(\mathrm{R}=0.91$ in Fig. 5) implies that accurate low-cost estimation of $S I$ is possible in clinical real-time with the methods described in this article. This result was obtained based on well-founded inferences into the insulin concentrations in interstitial fluid obtained from fully sampled DIST tests with insulin and Cpeptide sampling. Dependable assumptions from the protocol diminish the effects of the error in developing the population parameter estimations. The resulting simulated insulin concentrations are sufficiently accurate to allow good confidence in the derived SI.

Although there is a marginal reduction in correlation when the derivation set is isolated from the validation set, a strong correlation still exists $(\mathrm{R}=0.88$ in Fig. 6). Two possible issues could be the cause of the slight fall in correlation from Fig. (5). First, when the DISTq solver estimates an individual's $S I$ using population relationships generated from the full subject data set, including data from this particular individual, the accuracy could be positively biased. This concern is allayed when considering that the relationship equations are smooth, and no attempt has been made to fit the population variability in the parameter estimation graphs shown in Fig. (3). Fitting this variation in the population parameter graphs would allow a stronger correlation in this study, but it would be at the expense of applicability of the DISTq method to the wider population.

The second, more likely reason for a loss in correlation is the increasing sparseness of the derivation or development population, which in some cases may be less indicative of the population as a whole. A larger derivation set of full DIST data is not yet available to resolve this issue, but would enable a more refined and conclusive result. Similarly, low dose IM-FSIVGTT data with full insulin and C-peptide sampling could be used with the model of Equations (1) to (5) to enable a larger data set to generate more representative population based insulin relationships shown in Equations (14) to (18).

In particular, the data used in this study was generated during the pilot study of the fully sampled DIST test and is inclusive of tests where the clinical protocol was under development and not necessarily consistent. Furthermore, the dosing level is not consistent as varied dosing levels were part of the pilot study [17]. It is expected that varying the dose would have a proportional effect on $U_{e n}$. However, upon visual inspection of the relevant relationship graphs in Fig. (3), this expectation did not seem to be consistently the case. There is also a concern that saturation effects at different dosing levels may affect the value of liver clearance $n_{L}$. Again, visual inspection did not show any significant difference in this case. If sufficient data becomes available with a set protocol and dosing regime, the parameter estimation stage would be stronger and could be fortified with confidence bounds to give a recommendation of the confidence interval of the DISTq derived SI. Such studies are ongoing but data has not yet become available.

\subsection{Comparison to Other Tests}

The performance of any new test must also be assessed in light of tests of equivalent intensity. The intensity of the DISTq is greater than HOMA, and, in terms of clinician activity, the 2-hr Oral Glucose Tolerance Test (2hr-OGTT), in which an oral load of glucose is ingested and the glucose level is sampled after two hours. In contrast to the HOMA and 2 hr-OGTT, which only require a nurse to perform, the DISTq protocol requires a more clinical environment with either nurses or doctors with experience in giving intravenous insulin, who can respond to hypoglycaemia if necessary. The DISTq protocol takes 30 - 45 minutes to perform and includes blood samples every 5 to 10 minutes, which is of greater clinical intensity than the $2 \mathrm{hr}$-OGTT, which only requires one (sometimes two) blood samples. Thus, to be considered as a viable clinical option, the DISTq must predict $S I$, as well as changes in $S I$ in response to therapy or intervention, with greater accuracy and reliability than either the HOMA or 2hr-OGTT.

The 2hr-OGTT has correlated well to the euglycaemic clamp, achieving $\mathrm{R}=0.74$ [16]. This result is in contrast to a number of studies, which show relatively low repeatability in terms of re-classifying NGT, IGT and T2DM subjects (50$65 \%$ repetition) $[7,10,34]$. In contrast, Fig. (7) shows DIST and DISTq should capture changes in SI much better than HOMA, and would likely have similar performance compared to the OGTT as noted above.

The DISTq differs compared to the 2hr-OGTT by the approach in which insulin and glucose are elevated in the test subject. Insulin is not measured in either the 2hr-OGTT or DISTq. However, the DISTq protocol introduces a known bolus of insulin at a known time, which is not done in the 2hr-OGTT. This bolus accounts for the majority of the subjects' plasma insulin and allows significantly more accurate predictions of the insulin concentration that are not possible in the $2 \mathrm{hr}$-OGTT protocol, which relies only on an endogenous response to a larger glucose challenge. The endogenous insulin response is much more varying within individuals and is harder to predict. In addition, the $2 \mathrm{hr}-$ OGTT protocol primarily measures the ability to dispose of glucose, and the efficiency of insulin is not assessed. Thus, the derived metric is not a fully true reflection of SI, but it is instead an overall measure of the ability to dispose of glucose. The $2 \mathrm{hr}$-OGTT metric is thus effective in some clinical scenarios, such as in diagnosing individuals with impaired glucose tolerance (IGT) or type 2 diabetes (T2DM). However, it is not suitable in studies where accurate assessment of insulin sensitivity, or modest changes in this value over time or therapy are critical, such as when monitoring the patient response to drug therapy. Thus the $2 \mathrm{hr}-\mathrm{OGTT}$ is an accepted crude T2DM diagnostic test, but not effective in tracking or quantifying insulin sensitivity changes.

The HOMA is a simple protocol requiring only one blood sample analysed for glucose and insulin. A simple linear 
formula then calcualtes an individuals insulin resistance level [18]. However, this simplicity means that the derived metric is not representative of insulin mediated glucose disposal in a dynamic, non-fasting situation. The pulsatile nature of insulin secretion $[31,35]$ and lag in the insulinglucose dynamics cause effectively random errors in the derived HOMA metric. The relatively poor correlation of HOMA to the euglycaemic clamp SI metric $\left(\mathrm{ISI}_{\mathrm{G}}\right)(\mathrm{R}=-0.37)$ found in this study emphasises the uncertainty created by these fluctuations. Furthermore, Fig. (7) shows that HOMA has a clear inability to capture modest changes in $S I$ due to intervention and therapy. The $S I_{D I S T q}$ derived in the Monte Carlo study accurately repeated the clinically observed intervention-driven intra-patient change in $\mathrm{ISI}_{\mathrm{G}}$ with a correlation of $\mathrm{R}=0.92$. In contrast, the HOMA achieved a much lower $\mathrm{R}=-0.22$ correlation. These results indicate that the DISTq approach should be a better and more effective test in presence of the physiological and clinically induced variations accounted for in the Monte Carlo study.

This strength can be largely attributed to the pacifying effect that a longitudinal intervention study has on the DISTq sub-process that introduces greatest variability. In particular, the primary cause of variability in $S I_{D I S T q}$ is the population-based parameter estimation stage (Equations (14) to (18)). The average error between the full DIST solved parameters and the parameters derived from Equations (14) to (18) is approximately $35 \%$. Although this value is significant, the effect on $S I$ is minimised by the specific structure of the DIST protocol and DISTq method. As changes in these estimated parameters are likely to be minimal during a longitudinal study between interventions, or if measured regularly over 3-12 month intervals, the change in SI can be defined by the changes in the gradient of glucose caused by the altered efficiency of the insulin bolus in the subject. Studies that could utilise DISTq include drug trials for enhancing insulin sensitivity and interventional dietary or lifestyle studies.

Although the prediction capability of the DISTq method exceeds that of tests with similar intensity, the CV of the derived metric is relatively high. The CV of $21 \%$ predicted by the Monte-Carlo simulation is higher than what would be expected in the $2 \mathrm{hr}$-OGTT or the HOMA. However, for diagnostic purposes this effect can be ameliorated. In particular, the stored blood samples from borderline diagnoses could be analysed for insulin and C-peptide and an $S I$ value found using the full DIST analysis. Thus, an SI value with a much greater certainty could be estimated $(\mathrm{CV}=4-6 \%)$ and the subject would not have to participate in another test. Although, this solution would eliminate the real-time capability of the test for cases with borderline DISTq results, it would only be necessary in limited cases needing more resolution.

\subsection{Clinical Impact, Issues and Limitations}

The area of significant clinical interest is the lower end of the $S I$ scale $\left(1.0-5.0 \mathrm{e}^{-4} \mathrm{~L} \cdot \mathrm{mU}^{-1} \cdot \mathrm{min}^{-1}\right)$, as most individuals with diabetes risk would fall into this range [24]. The CV of the DISTq is highest in this range due to a number of factors in the method and protocol. The small glucose disposal rate variations generated by the Monte Carlo simulation alter the SI predictions. In particular, small shifts in this $S I$ have a greater effect on the unknown parameter estimation subprocess as the equation gradients are highest in this range. The re-simulated insulin concentration profile would incline SI further in the direction of any noise generated change. This error and its propagation is unavoidable with this method. The higher gradients in this range are necessary to capture the rapid increase in pancreatic insulin output and basal insulin concentration found in some pre-diabetic states. However, again, any borderline diagnosis with a DISTq could result in a more accurate DIST being done with stored blood, as only the assays used are different between DIST and DISTq analyses. As a result, expensive insulin and Cpeptide assays are taken only where needed and a hierarchy of tests is enabled.

This variability in metabolic response to the test for subjects with low SI increases the parameter estimation uncertainty in the low SI region for the DISTq. The increase in error is partly a result of the nature of the natural progression of type 2 diabetes. A newly diagnosed type 2 diabetic individual can exhibit a low $S I$ with very high basal insulin concentrations and high second phase insulin secretion $\left(U_{e n}\right)$. In contrast, a long-term type 2 diabetic individual with the same or very similar SI may have either exhausted or significantly diminished beta cell function, resulting in very low basal and second phase insulin secretion levels. Hence, there are two very similar SI values with markedly different endogenous insulin responses depending on time of exposure to the disease. This characteristic can be observed as the "trumpet" shape seen in the parameter estimation graphs of Fig. (3) at a low SI. Accounting for these differences remains a topic of future research.

The clinical real-time capability of the DISTq may allow new applications to benefit from SI testing. Glycaemic control and sepsis diagnosis in an intensive care environment could be improved [19]. It may also aid the planning of glycaemic regulation for newly diagnosed diabetic individuals. Further research must be undertaken to ensure that the protocol is suitable, and that the population-based equations are relevant in these situations.

\section{CONCLUSIONS}

Accurate $S I$ estimation is possible in clinical real-time with only glucose samples using the DIST protocol and a number of population based parameter assumptions. There is a strong correlation between fully sampled $S I_{D I S T}$ and $S I_{D I S T q}$ of $\mathrm{R}=0.91$, which is maintained outside the parameter estimation population. The method is robust as the protocol avoids saturation characteristics and allows very good assumptions about an individual's insulin concentrations.

When choosing an insulin-sensitivity test the clinician or researcher should decide what level of resolution, intensity, cost, complexity, speed and confidence is best suited for that particular application or study. The DISTq is not an answer for all of these purposes, but should be considered when a low intensity, very inexpensive, but relatively accurate estimation of $S I$ is desired. The low cost and relatively low intensity for the accuracy and resolution delivered may allow greater numbers to be studied or tested. The test requires advanced mathematical processes, but once provided, the 
data can be analysed and delivered in clinical real-time and the researcher or clinician can thus get a result immediately.

\section{REFERENCES}

[1] Martin BC, Warram JH, Krolewski AS, Bergman R, Soeldner JS, Kahn CR. Role of glucose and insulin resistance in development of type 2 diabetes mellitus: results of a 25 -year follow-up study. Lancet 1992; 340(8825): 925-9.

[2] ADA. Economic consequences of diabetes mellitus in the U.S. in 1997. American Diabetes Association. Diabetes Care 1998; 21(2): 296-309.

[3] ADA. Intense blood glucose control yields no significant effect on CVD reduction in VA diabetes trial 2008.

[4] Ferrannini E, Mari A. How to measure insulin sensitivity. J Hypertens 1998; 16(7): 895-906.

[5] Pacini G, Mari A. Methods for clinical assessment of insulin sensitivity and beta-cell function. Best Pract Res Clin Endocrinol Metab 2003; 17(3): 305-22.

[6] DeFronzo RA, Tobin JD, Andres R. Glucose clamp technique: a method for quantifying insulin secretion and resistance. Am J Physiol 1979; 237(3): E214-23.

[7] Levy JC, Morris RJ, Hammersley M, Turner RC. Discrimination, adjusted correlation, and equivalence of imprecise tests: application to glucose tolerance. Am J Physiol 1999; 276 (2 pt 1): E365-75.

[8] Galvin P, Ward GM, Walters JM, et al. A simple method for quantitation of insulin sensitivity and insulin release form an intravenous glucose tolerance test. Diabet Med 1992; 9(10): 921-8.

[9] Bonora E, Targher G, Alberiche M, et al. Homeostasis model assessment closely mirrors the glucose clamp technique in the assessment of insulin sensitivity. Diabetes Care 2000; 23(1): 5763.

[10] Ko GTC, Chan JCN, Woo J, et al. The reproducibility and usefulness of the oral glucose tolerance test in screening for diabetes and other cardiovascular risk factors. Ann Clin Biochem 1998; 35: 62-7.

[11] Monzillo LU, Hamdy O. Evaluation of insulin sensitivity in clinical practice and in research settings. Nutr Rev 2003; 61(12): 397-412.

[12] Foley JE, Chen YD, Lardinois CK, Hollenbeck CB, Liu GC, Reaven GM. Estimates of in vivo insulin action in humans: comparison of the insulin clamp and the minimal model techniques. Horm Metab Res 1985; 17(8): 406-9.

[13] Bergman RN, Prager R, Volund A, Olefsky JM. Equivalence of the insulin sensitivity index in man derived by the minimal model method and the euglycemic glucose clamp. J Clin Invest 1987; 79(3): 790-800.

[14] Stumvoll M, Mitrakou A, Pimenta W, et al. Use of the oral glucose tolerance test to assess insulin release and insulin sensitivity. Diabetes Care 2000; 23(3): 295-301.

[15] Matsuda M, DeFronzo RA. Insulin sensitivity indices obtained from oral glucose tolerance testing: comparison with the euglycemic insulin clamp. Diabetes Care 1999; 22(9): 1462-70.

[16] Ferrannini E, Gastaldelli A, Miyazaki Y, Matsuda M, Mari A, DeFronzo RA. beta-Cell function in subjects spanning the range from normal glucose tolerance to overt diabetes: a new analysis. J Clin Endocrinol Metab 2005; 90(1): 493-500.

[17] Lotz T, Chase JG, McAuley KA, et al. Monte Carlo analysis of a new model-based method for insulin sensitivity testing. Comput Methods Programs Biomed 2008; 89: 215-25.
[18] Matthews DR, Hosker JP, Rudenski AS, Naylor BA, Treacher DF, Turner RC. Homeostasis model assessment: insulin resistance and beta-cell function from fasting plasma glucose and insulin concentrations in man. Diabetologia 1985; 28(7): 412-9.

[19] Blakemore A, Wang SH, Le Compte AJ, et al. Model-based insulin sensitivity as a sepsis diagnostic in critical care. J Diabetes Sci Technol 2008; 2(3): 468-77.

[20] McAuley KA, Williams SM, Mann JI, et al. Intensive lifestyle changes are necessary to improve insulin sensitivity: a randomized controlled trial. Diabetes Care 2002; 25(3): 445-52.

[21] Stumvoll M, Nurjhan N, Perriello G, Dailey G, Gerich JE. Metabolic Effects of Metformin in Non-Insulin-Dependent Diabetes Mellitus. N Engl J Med 1995; 333(9): 550-54.

[22] Zimmet P, Boyko EJ, Collier GR, deCourten M. Etiology of the metabolic syndrome: Potential role of insulin resistance, leptin resistance, and other players. Ann N Y Acad Sci 1999; 892: 25-44.

[23] Hanley AJ, Williams K, Festa A, Wagenknecht LE, D'Agostino RB, Jr., Haffner S. Liver markers and development of the metabolic syndrome: The insulin resistance atherosclerosis study. Diabetes 2005; 54(11): 3140-7.

[24] Lotz T. High resolution clinical model-based assessment of insulin sensitivity. Christchurch: University of Canterbury 2007.

[25] Carson ER, Cobelli C. Modelling methodology for physiology and medicine. San Diego: Academic Press 2001.

[26] Ferrannini E, Cobelli C. The kinetics of insulin in man. I. General aspects. Diabetes Metab Rev 1987; 3(2); 335-63.

[27] Van Cauter E, Mestrez F, Sturis J, Polonsky KS. Estimation of insulin secretion rates from C-peptide levels. Comparison of individual and standard kinetic parameters for C-peptide clearance. Diabetes 1992; 41(3): 368-77.

[28] Youssef IK, El-Arabawy HA. Picard iteration algorithm combined with Gauss-Seidel technique for initial value problems. Appl Math Comput 2007; 190(1): 345-55.

[29] Kim C, Kim SB. Modelling contaminant transport in a three-phase groundwater system with the Freundlich-type retardation factor. Environ Technol 2007; 28(2): 205-16.

[30] Hann CE, Chase JG, Lin J, Lotz T, Doran CV, Shaw GM. Integralbased parameter identification for long-term dynamic verification of a glucose-insulin system model. Comput Methods Programs Biomed 2005; 77(3); 259-70.

[31] Meier JJ, Veldhuis JD, Butler PC. Pulsatile insulin secretion dictates systemic insulin delivery by regulating hepatic insulin extraction in humans. Diabetes 2005; 54(6): 1649-56.

[32] Toffolo G, Campioni M, Basu R, Rizza RA, Cobelli C. A minimal model of insulin secretion and kinetics to assess hepatic insulin extraction. Am J Physiol Endocrinol Metab 2006; 290(1): E169E76.

[33] Ferrannini E, Cobelli C. The kinetics of insulin in man. II. Role of the liver. Diabetes Metab Rev 1987; 3(2); 365-97.

[34] Park PJ, Griffin S, Duffy SW, Wareham NJ. The effect of varying the screening interval on false positives and duration of undiagnosed disease in a screening programme for type 2 diabetes. J Med Screen 2000; 7(2): 91-6.

[35] Zarkovic M, Ciric J, Stojanovic M, et al. Effect of insulin sensitivity on pulsatile insulin secretion. Eur J Endocrinol 1999; 141(5): 494-501. 